\title{
Signal Decomposition for X-ray Dark-field Imaging
}

\author{
Sebastian Kaeppler ${ }^{1}$, Florian Bayer ${ }^{2}$, Thomas Weber $^{2}$, Andreas Maier ${ }^{1}$, \\ Gisela Anton ${ }^{2}$, Joachim Hornegger ${ }^{1}$, Matthias Beckmann ${ }^{3}$, Peter A. Fasching ${ }^{3}$, \\ Arndt Hartmann ${ }^{3}$, Felix Heindl ${ }^{3}$, Thilo Michel$^{2}$, Gueluemser Oezguel ${ }^{3}$, \\ Georg Pelzer ${ }^{2}$, Claudia Rauh ${ }^{3}$, Jens Rieger ${ }^{2}$, Ruediger Schulz-Wendtland ${ }^{3}$, \\ Michael Uder ${ }^{3}$, David Wachter ${ }^{3}$, Evelyn $W^{2} k_{k e l}{ }^{3}$, and Christian Riess ${ }^{1}$ \\ 1 Pattern Recognition Lab \\ 2 Erlangen Centre for Astroparticle Physics \\ 3 University Hospital of Erlangen \\ Friedrich-Alexander-University Erlangen-Nuremberg, Erlangen, Germany \\ sebastian.kaeppler@fau.de
}

\begin{abstract}
Grating-based X-ray dark-field imaging is a new imaging modality. It allows the visualization of structures at micrometer scale due to small-angle scattering of the X-ray beam. However, reading darkfield images is challenging as absorption and edge-diffraction effects also contribute to the dark-field signal, without adding diagnostic value. In this paper, we present a novel - and to our knowledge the first - algorithm for isolating small-angle scattering in dark-field images, which greatly improves their interpretability. To this end, our algorithm utilizes the information available from the absorption and differential phase images to identify clinically irrelevant contributions to the dark-field image. Experimental results on phantom and ex-vivo breast data promise a greatly enhanced diagnostic value of dark-field images.
\end{abstract}

\section{Introduction}

Absorption X-ray imaging is the standard modality for a wide range of applications. Recently, phase-sensitive X-ray imaging attracted much attention. Different measurement principles have been proposed to obtain phase-sensitive images, most notable are propagation-based systems [1], diffraction-enhanced systems [2] and grating-based interferometers [3]. One particular benefit of grating-based systems is that three output images are obtained, containing per pixel complementary information on absorption (i.e., overall intensity attenuation), differential phase shift of the X-ray wavefront, and dark-field (i.e., the contrast reduction of the grating pattern due to the object).

While the differential phase shift contrast (DPC) can significantly increase soft-tissue contrast over absorption imaging [4], the contrast loss of the grating pattern, referred to as dark-field signal (DFI), reveals unique information about structural tissue variations at micro and nano meter scale [5], often subsumed 


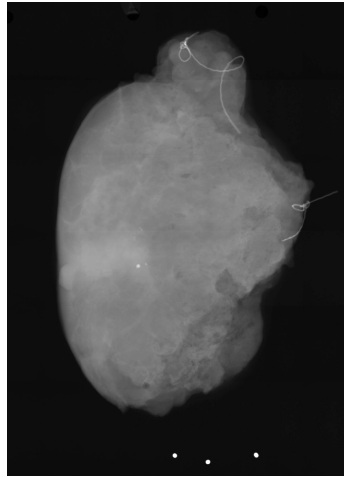

(a)

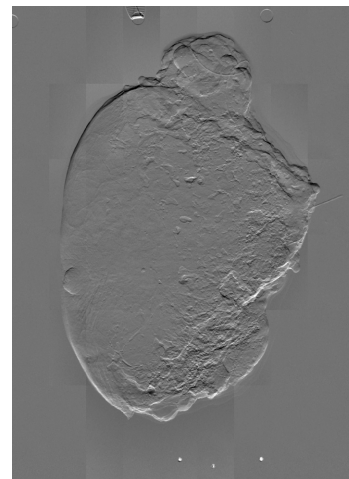

(b)

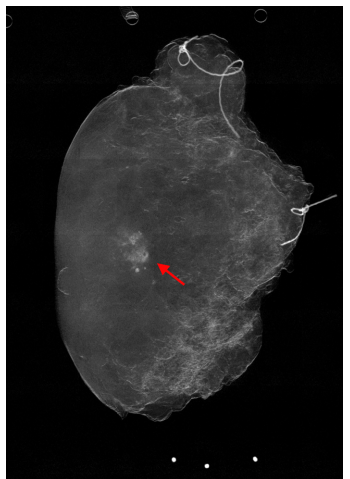

(c)

Fig. 1: Images of a cancerous mastectomy sample acquired using X-ray interferometry. (a) Absorption image. (b) Differential phase image. (c) Dark-field image. Note the visualization of the tumor in the dark-field image (red arrow).

as small-angle scattering. Such structures are well below the resolution limit of conventional X-ray imaging systems, and hence difficult to detect. First studies concluded that DFI yields important insights, and might be particularly useful for detecting microcalcifications in mammography (see, e.g., [6]).

However, for medical purposes, interpreting DFI images is difficult, as not only small structure variations contribute to the dark-field image. While its image formation process has not been fully understood yet, it has been empirically observed that scattering and beam hardening effects within the object create an absorption-like contribution to the dark-field signal. Additionally, diffraction effects at material edges also produce a dark-field signal. Fig. 1 shows an example for the absorption, differential-phase and dark-field image of a cancerous mastectomy sample. Note that the microcalcifications, although present in DFI, appear within a large amount of disturbing structural information.

In this work, we propose a method to greatly enhance the interpretability of dark-field images by removing or weakening these disturbing effects from the DFI image. For this task, we exploit the observations that a) disturbing influences in the dark-field signal often arise from the absorption or phase signal, and are hence correlated between these images, while b) small-angle scattering is contained only in the dark-field signal. We demonstrate on mammographic data that the result of this separation is a clean dark-field signal, in which microcalcifications can be readily detected.

Previous work on the post-processing of data obtained using interferometric $\mathrm{X}$-ray imaging is scarce and has only dealt with image fusion in combination with denoising [7] or visualization [8]. To our knowledge, this is the first algorithm which allows to isolate the unique small-angle scattering information contained within dark-field X-ray images. 


\section{Methods}

As no complete physical model of the dark-field image formation process is available, we assume three independent contributions to the dark-field image: absorption-based effects, such as scattering and beam hardening; edge diffraction and small-scale structure variations. Since only the signal due to the smallscale structure variations is unique to the DFI signal (and, for our application, a strong indicator of breast microcalcifications), we seek to remove the other two contributions by decorrelating the DFI image with the absorption and DPC signal. First, the absorption-based contribution is estimated and subtracted. In a second stage, signals due to edge diffraction effects are suppressed.

\subsection{Absorption correction}

In a first examination of the images, we noted that most unwanted DFI signals occur due to absorption effects. To remove these influences, we propose a method that is inspired by water correction for CT-reconstruction. There, a polynomial correction factor is commonly used to rescale the recorded intensities to account for the effects of scatter in homogeneous materials. For our task, we assume that there exists a transfer function $f_{\text {abso }}$ which maps pixel intensities of the absorption image to their corresponding contribution to the dark-field image. We further assume that this function is constant over the image (and thus for all materials in the object). We model this function as a polynomial of degree $p$. Let $\boldsymbol{A}$ be the absorption image and $\boldsymbol{D}$ be the dark-field image. We obtain a first estimate $f_{\text {abso }}^{0}$ of the transfer function by solving the least-squares problem

$$
f_{\text {abso }}^{0}=\underset{f_{\text {abso }}}{\arg \min }\left\|f_{\text {abso }}(\boldsymbol{A})-\boldsymbol{D}\right\|_{2}^{2} .
$$

This global estimation is only valid if - as initially observed - most of the DFI signal co-occurs in the absorption image. Still, the initial estimate is biased by the pixels of the DFI images which contain signals due to edge diffraction and structure variations. To address this issue, the estimates of $f_{\text {abso }}$ are iteratively refined. At each refinement step $i$, a thresholding map $\boldsymbol{T}^{i}$ is computed as

$$
\boldsymbol{T}^{i}(n)=\left\{\begin{array}{ll}
1 & \text { if } f_{\text {abso }}^{i}(\boldsymbol{A}(n)-\boldsymbol{D}(n))<\left(t_{\text {thresh }} \cdot \max (\boldsymbol{D})\right) \\
0 & \text { otherwise }
\end{array} .\right.
$$

The threshold operator selects pixels of the dark-field image that arise only from absorption effects for estimating the transfer function. Refinements of the transfer function $f_{\text {abso }}^{i}, 1 \leq i \leq i_{\max }$ are estimated by

$$
f_{\text {abso }}^{i+1}=\underset{f_{\text {abso }}}{\arg \min }\left\|\boldsymbol{T}^{i} \cdot\left(f_{\text {abso }}(\boldsymbol{A})-\boldsymbol{D}\right)\right\|_{2}^{2} .
$$

Finally, the absorption-corrected dark-field image $\boldsymbol{D}_{\text {abso }}$ is computed as

$$
\boldsymbol{D}_{\text {abso }}=\boldsymbol{D}-\max \left(f_{\text {abso }}^{i_{\max }}(\boldsymbol{A}), 0\right),
$$

where the maximum operator ensures the non-negativity of the estimated absorption contribution. An example of the absorption correction is shown in Fig. 2 . 


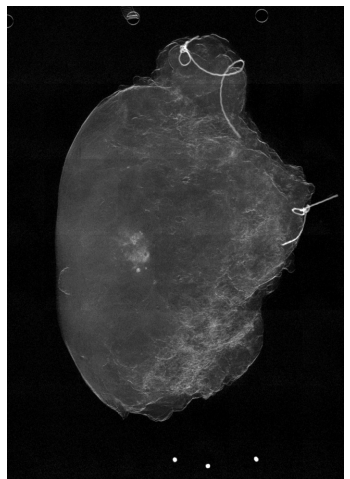

(a)

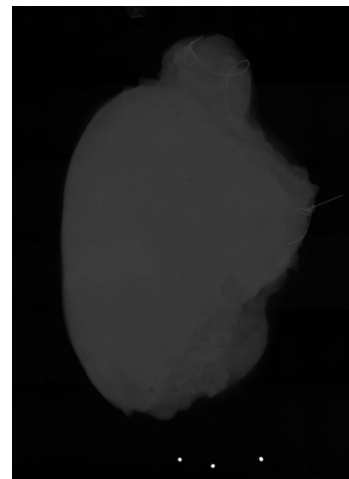

(b)

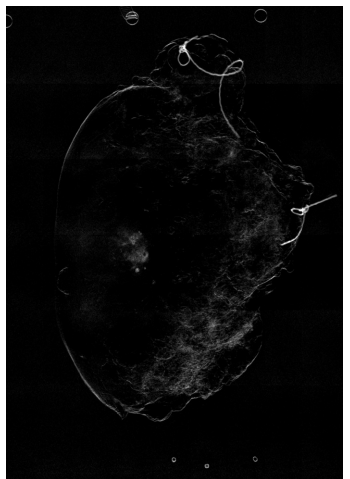

(c)

Fig. 2: Example of the absorption correction. (a) Input dark-field image $\boldsymbol{D}$. (b) Estimated absorption contribution $f_{\text {abso }}^{i_{\max }}(\boldsymbol{A})$. (c) Absorption-corrected darkfield image $\boldsymbol{D}_{\text {abso }}$.

\subsection{Edge diffraction correction}

After obtaining the absorption-corrected dark-field image $\boldsymbol{D}_{\text {abso }}$, the second stage of our algorithm attempts to correct for the signal contribution due to diffraction effects at object edges. We utilize the fact that the differential phase image $\boldsymbol{P}$ shows a significantly increased edge contrast compared to the absorption image. Thus, we assume that a signal $\boldsymbol{D}(n)$ at a pixel $n$ is likely due to edge diffraction if the corresponding magnitude of the DPC image, $\operatorname{abs}(\boldsymbol{P}(n))$ is high. We've experimented with several methods about how to utilize this information. Using the information of the DPC image directly is difficult due to scaling variations of individual edges. Local methods, such as cross-correlation maps, suffer from noise amplification problems, as the dark field image typically shows a low signal-to-noise ratio. We found that Independent Component Analysis (ICA) is a useful preprocessing step of the edge information. To this end, we compute the two resulting independent components of the absorption-corrected dark-field image and the absolute value, $\operatorname{abs}(\boldsymbol{P})$, of the differential phase image. To obtain the independent components we utilize the FastICA algorithm [9], using the deflation method with the tanh function as nonlinearity measurement. Only one of the two independent components $\boldsymbol{I}_{1}$ and $\boldsymbol{I}_{2}$ corresponds to the edge map. Additionally, the data scaling after ICA can be arbitrary. Thus we compute rescaled components $\boldsymbol{I}_{1}^{R}$ and $\boldsymbol{I}_{2}^{R}$ to fit the DPC image in a least squares sense:

$$
\boldsymbol{I}_{j}^{R}=\underset{a, b}{\arg \min }\left\|\left(a \cdot \boldsymbol{I}_{j}+b\right)-\operatorname{abs}(\boldsymbol{P})\right\|_{2}^{2} .
$$

We then select the component as edge map $\boldsymbol{E}$ that fits the DPC image best:

$$
\boldsymbol{E}=\left\{\begin{array}{ll}
\max \left(\boldsymbol{I}_{1}^{R}, 0\right) & \text { if }\left\|\boldsymbol{I}_{1}^{R}-\operatorname{abs}(\boldsymbol{P})\right\|_{2}<\left\|\boldsymbol{I}_{2}^{R}-\operatorname{abs}(\boldsymbol{P})\right\|_{2} \\
\max \left(\boldsymbol{I}_{2}^{R}, 0\right) & \text { otherwise }
\end{array} .\right.
$$




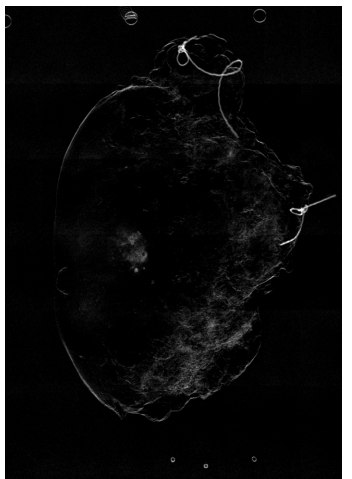

(a)

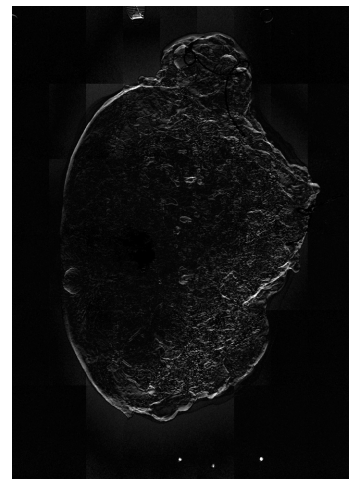

(b)

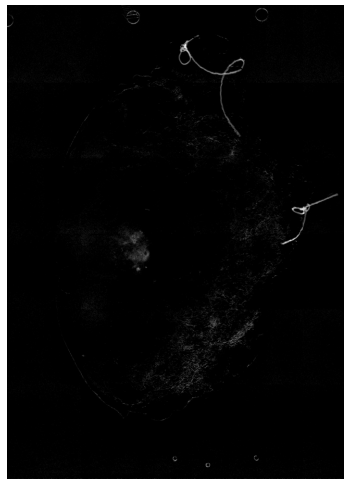

(c)

Fig. 3: Example of the edge correction. (a) Absorption-corrected dark-field image $\boldsymbol{D}_{\text {abso }}$. (b) Computed edge map $\boldsymbol{E}$. (c) Final image $\boldsymbol{D}_{\text {corr }}$. Edge contributions are well suppressed, except for the specimen cutting edge at the bottom right.

Again, we enforce the non-negativity of the edge map. After determining the edge map, the edge- and absorption-corrected dark-field image $\boldsymbol{D}_{\text {corr }}$ is computed using soft shrinkage

$$
\boldsymbol{D}_{\text {corr }}=\max \left(\boldsymbol{D}_{\text {abso }}-t_{\text {shrink }} \cdot \boldsymbol{E}, 0\right) .
$$

The shrinkage factor $t_{\text {shrink }}$ can be used to tune the balance between edge removal and signal preservation. Finally, negative intensities are set to zero and the resulting image is normalized to the interval $[0 ; 1]$ for the purpose of visualization. Fig. 3 illustrates the edge removal step.

\section{Evaluation and Results}

Evaluation of our processing scheme is challenging. As the DFI formation is not yet fully understood, it is difficult to obtain accurate simulations that include the absorption and edge-diffraction effects that we seek to remove. Thus, we were not able to use synthetic evaluation data. Additionally, performing a reader study is non-trivial, as radiologists are not yet familiar with the reading of darkfield images. Hence, we evaluate our approach in two parts. First, we present a quantitative evaluation of the algorithm on a phantom. Second, we display qualitative results of applying our algorithm to cancerous mastectomy samples. All images were acquired using our experimental setup of a three-grating TalbotLau interferometer. Details on the setup are reported in [6]. As no ground truth data is available to optimize the parameters of our algorithm, we determining them heuristically. To this end, we used an extensive database of 76 data sets, comprising various objects. For the absorption correction, we set the polynomial 


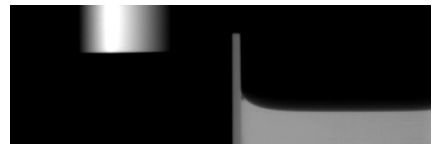

(a)

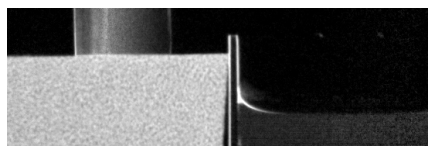

(c)

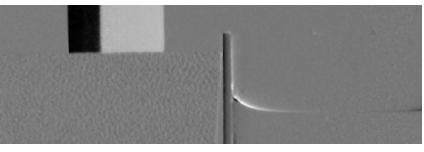

(b)

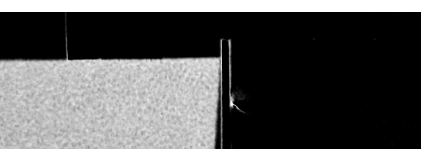

(d)

Fig. 4: The test phantom consisting of a teflon wedge on top of a foam block, next to a basin filled with water. (a) Absorption image. (b) Differential phase image. (c) Dark-field image. (d) Processed dark-field image.

degree $p=4$, the number of iterations $i_{\max }=2$ and the threshold $t_{\text {thresh }}=0.05$. For the edge correction, we set the shrinkage factor $t_{\text {shrink }}=1.0$.

\subsection{Phantom data}

The test phantom for the quantitative evaluation, depicted in Fig. 4, consisted of a foam block with a teflon wedge on top (left side) and a basin filled with water (right side). As expected, the foam block is invisible in the absorption image, yet is clearly visible on the dark-field image due to its porous structure causing small-angle scattering. The water and the wedge also show some darkfield signal due to absorption effects. After processing with our algorithm, the foam block remains clearly visible, while the signals of the wedge and the water are suppressed.

For quantitative evaluation, we calculated the correlation of the intensities between the original and the processed dark-field image within and outside of the foam block. For the pixels belonging to the foam block, the correlation is 0.946. For the outside pixels, the correlation is 0.588 . In addition to the correlation coefficient, we computed the average contrast between the foam block and the other two objects before and after processing. The contrast increased from $5.23: 1$ in the unprocessed image to $46.56: 1$ in the processed image.

\subsection{Mastectomy data}

For qualitative evaluation, we examined 12 cancerous mastectomic specimen. In Fig. 5, we present two representative examples. Visual inspection of the resulting processed images reveals that absorption-based contributions are effectively suppressed. Within the specimens, edge effects have been almost completely removed. At the border of the objects, especially at the cutting edges, some residual edge signals remain. Signals due to small-angle scattering caused by microcalcification at the tumors have been well preserved. Also, the marking strings remain 

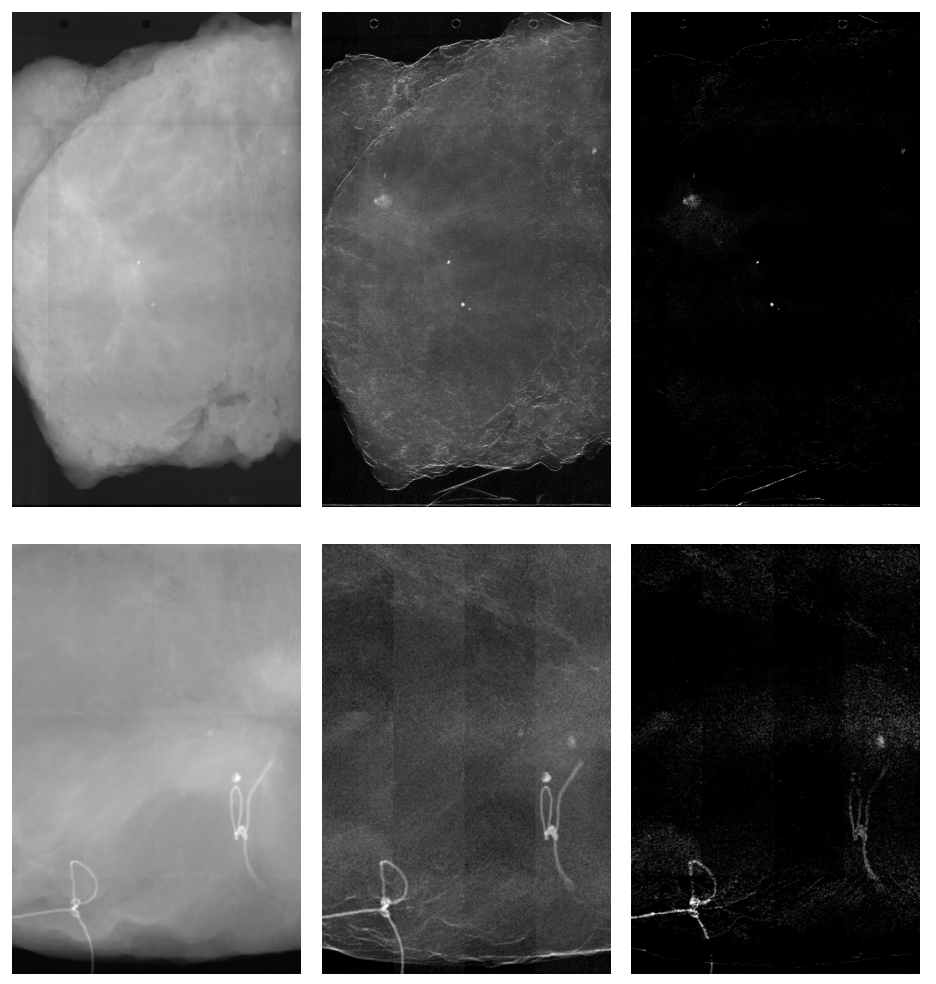

Fig. 5: Two additional examples of cancerous mastectomy samples processed using the proposed algorithm. From left to right: absorption, dark-field and processed dark-field images. Note the improved differentiability between the tumor and surrounding tissue.

visible in the dark-field image due to their fiber structure. However, we notice some loss of signal at pixel-level. We attribute this to noise in the dark-field signal and our edge map used for shrinking the signal.

\section{Discussion and Conclusions}

We presented an algorithm to determine the influence of small-scale structure variations to dark-field images, using a two-step decorrelation approach. To our knowledge, this is the first algorithm proposed for this purpose. We have shown a quantitative evaluation using a test phantom and a qualitative evaluation using mastectomic breast images. Overall, we observe a good suppression of absorption- and phase-related signals in the DFI image. The resulting images have the potential to provide greatly enhanced diagnostic value for, e.g., early detection of breast cancer lesions and DFI interpretation in general. 
Possibilities for future research are vast. Due to broadening of the edge responses in the dark-field image, some edge diffraction effects are not fully removed. A physically or learning-based edge model could account for this effect. Also, we currently do not utilize prior information about the structure of the dark-field signal, which could be used to devise more effective task-specific algorithms. Finally, we aim to perform a radiologist reader study to assess the clinical impact of the proposed algorithm.

\section{Acknowledgments}

This work was funded by the German Ministry for Education and Research (BMBF), project grant No. 13EX1212B and the cluster of excellence Medical Valley EMN and Siemens Healthcare. The authors thank Dr. Jürgen Mohr and Jan Meiser from the Karlsruhe Institute of Technology and the Karlsruhe Nano Micro Facility (KNMF) for manufacturing the gratings used in the experiments. Patient consent and approval of the local ethics committee were obtained before the experiments involving the breast samples.

\section{References}

1. Wilkins, S.W., Gureyev, T.E., Gao, D., Pogany, A., Stevenson, A.W.: Phasecontrast imaging using polychromatic hard x-rays. Nature 384(6607) (November 1996) 335-338

2. Parham, C., Zhong, Z., Connor, D.M., Chapman, L.D., Pisano, E.D.: Design and implementation of a compact low-dose diffraction enhanced medical imaging system. Academic Radiology 16(8) (August 2009) 911-917

3. Pfeiffer, F., Weitkamp, T., Bunk, O., David, C.: Phase retrieval and differential phase-contrast imaging with low-brilliance x-ray sources. Nature Physics 2(4) (April 2006) 258-261

4. Momose, A., Yashiro, W., Takeda, Y.: Sensitivity of x-ray phase imaging based on talbot interferometry. Japanese Journal of Applied Physics 47(10) (October 2008) 8077-8080

5. Yashiro, W., Terui, Y., Kawabata, K., Momose, A.: On the origin of visibility contrast in x-ray talbot interferometry. Optics express 18(16) (August 2010) 1689016901

6. Michel, T., Rieger, J., Anton, G., Bayer, F., Beckmann, M., Durst, J., Fasching, P., Haas, W., Hartmann, A., Pelzer, G., Radicke, M., Rauh, C., Ritter, A., Sievers, P., Schulz-Wendtland, R., Uder, M., Wachter, D., Weber, T., Wenkel, E., Zang, A.: On a dark-field signal generated by micrometer-sized calcifications in phase-contrast mammography. Physics in Medicine and Biology 58(8) (April 2013) 2713-2732

7. Wang, Z., Clavijo, C.A., Roessl, E., van Stevendaal, U., Koehler, T., Hauser, N., Stampanoni, M.: Image fusion scheme for differential phase contrast mammography. Journal of Instrumentation 8(07) (2013) C07011

8. Haas, W., Polyanskaya, M., Bayer, F., Gödel, K., Hofmann, H., Rieger, J., Ritter, A., Weber, T., Wucherer, L., Durst, J., Michel, T., Anton, G., Hornegger, J.: Image fusion in x-ray differential phase-contrast imaging. In: SPIE Medical Imaging 2012. (February 2012) 83143U

9. Hyvarinen, A.: Fast and robust fixed-point algorithms for independent component analysis. IEEE Transactions on Neural Networks 10(3) (1999) 626-634 